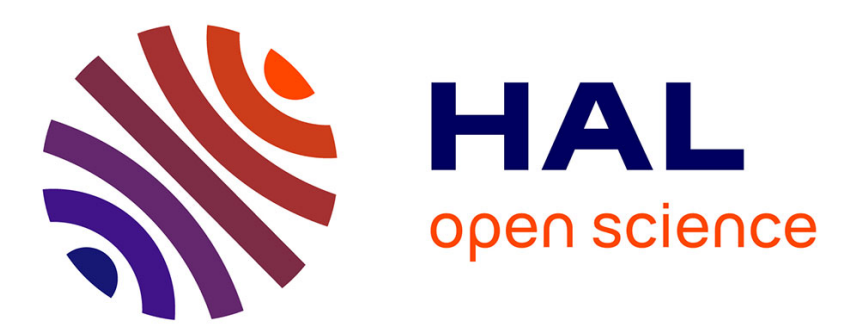

\title{
Dosimetry for cell irradiation using orthovoltage (40-300 kv) x-ray facilities
}

Morgane dos Santos, Vincent Paget, Francois Trompier, Gaetan Gruel, Fabien Milliat

\section{> To cite this version:}

Morgane dos Santos, Vincent Paget, Francois Trompier, Gaetan Gruel, Fabien Milliat. Dosimetry for cell irradiation using orthovoltage $(40-300 \mathrm{kv})$ x-ray facilities. Journal of visualized experiments : JoVE, 2021, 2021 (168), pp.e61645. 10.3791/61645 . hal-03207090

\section{HAL Id: hal-03207090 https://hal.science/hal-03207090}

Submitted on 23 Apr 2021

HAL is a multi-disciplinary open access archive for the deposit and dissemination of scientific research documents, whether they are published or not. The documents may come from teaching and research institutions in France or abroad, or from public or private research centers.
L'archive ouverte pluridisciplinaire HAL, est destinée au dépôt et à la diffusion de documents scientifiques de niveau recherche, publiés ou non, émanant des établissements d'enseignement et de recherche français ou étrangers, des laboratoires publics ou privés. 


\section{Journal of Visualized Experiments \\ Dosimetry for cell irradiation using orthovoltage (40-300 kV) X-ray facilities --Manuscript Draft--}

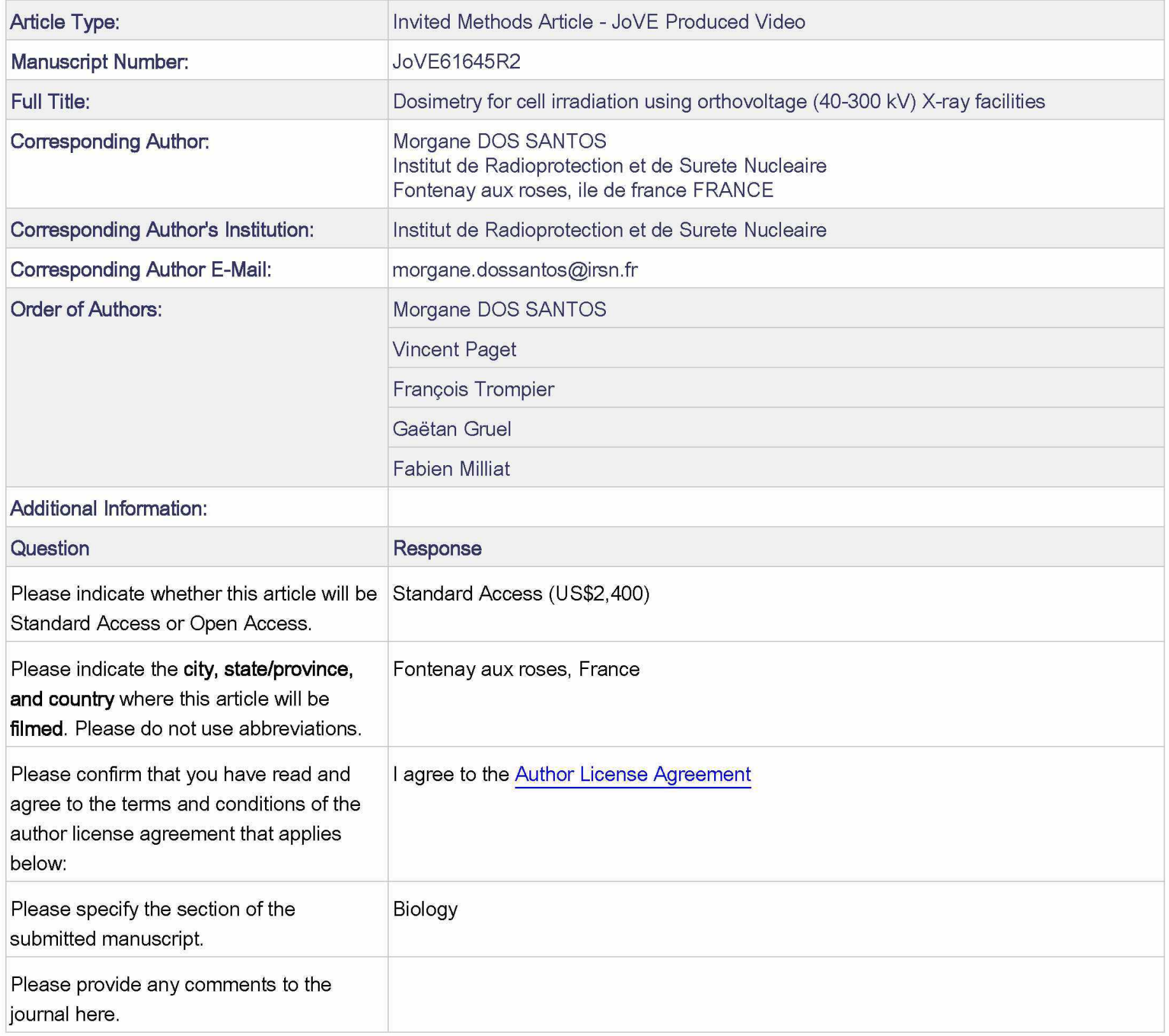




\section{Standard Manuscript Template}

Please Remove all Gray Text before Submitting
1

TITLE:

Dosimetry for Cell Irradiation Using Orthovoltage (40-300 kV) X-Ray Facilities

\section{AUTHORS \& AFFILIATIONS:}

Morgane Dos Santos ${ }^{1}$, Vincent Paget ${ }^{2}$, François Trompier ${ }^{3}$, Gaëtan Gruel ${ }^{1}$, Fabien Milliat ${ }^{2}$

${ }^{1}$ Department of RAdiobiology and Regenerative MEDicine (SERAMED), Laboratory of Radiobiology of Accidental Exposures (LRAcc), Institute for Radiological Protection and Nuclear Safety (IRSN), Fontenay-aux-Roses, France

${ }^{2}$ Department of RAdiobiology and Regenerative MEDicine (SERAMED), Laboratory of MEDical Radiobialogy (LRMed), Institute for Radiological Protection and Nuclear Safety (IRSN), Fontenayaux-Roses, France

${ }^{3}$ Department of DOSimetry (SDOS), Ionizing Radiation Dosimetry Laboratory (LDRI), Institute for Radiological Protection and Nuclear Safety (IRSN), Fontenay-aux-Roses, France

Corresponding Author:

Morgane Dos Santos

(morgane.dossantos@irsn.fr)

Email Addresses of Co-authors:

François Trompier_ (francois.trompier@irsn.fr)

Vincent Paget

(vincent.paget@irsn.fr)

Gaëtan Gruel

(gaetan.gruel@irsn.fr)

Fabien Milliat

(fabien.milliat@irsn.fr)

\section{KEYWORDS:}

dosimetry, low energy X-rays, radiobiology, irradiation protocol, cell irradiation, X-ray facility

\section{SUMMARY:}

This document describes a new dosimetry protocol for cell irradiations using low energy X-ray equipment. Measurements are performed in conditions simulating real cell irradiation conditions as much as possible.

\section{ABSTRACT:}

The importance of dosimetry protocols and standards for radiobiological studies is self-evident. Several protocols have been proposed for dose determination using low energy $\mathrm{X}$-rays facilities, but depending on the irradiation configurations, samples, materials or beam quality, it is sometimes difficult to know which protocol is the most appropriate to employ. We, therefore, propose a dosimetry protocol for cell irradiations using low energy $\mathrm{X}$-rays facility. The aim of this method is to perform the dose estimation at the level of the cell monolayer to make it as close as possible to real cell irradiation conditions. The different steps of the protocol are as follows determination of the irradiation parameters (high voltage, intensity, cell container etc.), determination of the beam quality index (high voltage-half value layer couple), dose rate measurement with ionization chamber calibrated in air kerma conditions, quantification of the 
attenuation and scattering of the cell culture medium with EBT3 radiochromic films and determination of the dose rate at the cellular level. This methodology must be performed for each new cell irradiation configuration as the modification of only one parameter can strongly impact the real dose deposition at the level of the cell monolayer, particularly involving low energy X-rays.

\section{INTRODUCTION:}

The aim of radiobiology is to establish links between the delivered dose and the biological effects, dosimetry is a crucial aspect in the design of radiobiological experiments. For over 30 years, the importance of dosimetry standards and the harmonization of practices have been highlighted ${ }^{1-5}$. To establish a dose rate reference, several protocols exist ${ }^{6-10}$, however, as shown by Peixoto and Andreo ${ }^{11}$, there can be differences of up to $7 \%$ depending on the dosimetric quantity used for the dose rate determination. Moreover, even if protocols exist, it is sometimes difficult to know which protocol is the most suitable for a particular application, if any, because the dose rate for the cells depends on parameters such as the cell container, quantity of cell culture media or beam quality, for example. The scattering and the backscattering for this type of irradiation is also a very important parameter to take into account. Indeed, for low and medium energy X-rays, in the AAPM TG-61 reference protocol ${ }^{10}$, the absorbed dose in water is measured at the surface of a water phantom. Taking into account the very specific cell irradiation conditions, small volume of cell culture media surrounded by air, that are therefore closer to kerma conditions, so in air, than those defined for absorbed dose with large water equivalent phantom as in TG-61 protocol. Therefore, we have chosen to use the kerma in water as dosimetric quantity for our reference rather than the absorbed dose in water. Thus, we are proposing a new approach to provide a better determination of the actual dose delivered to cells.

Moreover, another crucial aspect for radiobiological studies is the complete reporting of the methods and protocols used for irradiation in order to be able to reproduce, interpret and compare experimental results. In 2016, Pedersen et al. ${ }^{12}$ have highlighted the inadequate reporting of dosimetry in preclinical radiobiological studies. A larger recent study from Draeger et al. ${ }^{13}$ highlighted that even though some dosimetry parameters such as the dose, energy or source type are reported, a large part or physics and dosimetry parameters that are essential to properly replicate the irradiation conditions are missing. This large scale review, of more than 1,000 publications covering the past 20 years, shows a significant lack of the reporting of the physics and dosimetry conditions in radiobiological studies. Thus, a complete description of the protocol and the method utilized in radiobiological studies is mandatory in order to have robust and reproducible experiments.

Taking into account these different aspects, for the radiobiological experiments carried out at IRSN (Institute of Radiation Protection and Nuclear Safety), a stringent protocol was implemented for cell irradiations in an orthovoltage facility. This dosimetry protocol was designed in order to simulate the real cell irradiation conditions as much as possible and thus, to determine the actual dose delivered to cells. To this end, all the irradiation parameters are listed, and the beam quality index was evaluated by measuring the half value layer (HVL) for which some 
adaptations have been made as the standard recommendations from the AAPM protocol ${ }^{10}$ cannot be followed. The absolute dose rate measurement was then performed with the ionization chamber inside the cell container used for cell irradiations, and the attenuation and the scattering of the cell culture media was also quantified with EBT3 radiochromic films. As the modification of only one single parameter of the protocol can significantly impact the dose estimation, a dedicated dosimetry is performed for each cell irradiation configuration. Moreover, the HVL value must be calculated for each voltage-filter combination. In this present work, a voltage of $220 \mathrm{kV}$, an intensity of $3 \mathrm{~mA}$, and an inherent and an additional filtration of 0.8 and $0.15 \mathrm{~mm}$ of beryllium and copper, respectively, are used. The cell irradiation configuration chosen is on a T25 flask, where cells were irradiated with $5 \mathrm{~mL}$ of cell culture media.

\section{PROTOCOL:}

\section{Irradiation platform and determination of irradiation parameters}

1.1. Use an irradiation platform delivering low to medium energy X-rays. Determine the parameters of the experiment to ensure the robustness and the reproducibility of the radiobiological experiment: High voltage, Intensity, Filtration (inherent and additional), Half Value Layer (HVL), Effective energy, Detector used for dosimetry measurements, Source Sample Distance (SSD), Irradiation field (shape, size, geometry), Dosimetry quantity, Dosimetry method, Dose rate, Cell container and Quantity of cell culture media. All parameters used in this protocol are given in table 1.

\section{Beam quality index: Determination of the half value layer}

NOTE: The HVL is defined as the thickness of an attenuator (usually copper or aluminum) to reduce the intensity of the beam by a factor of two compared with the original value.

2.1. Set up the equipment (support, collimator, diaphragm, ionization) inside the irradiation enclosure by following the instructions in Figure 1. No attenuator material is used at this step.

2.2. Ensure that all the distances reported in Figure 1 are correct. Measure these with a tape measure.

2.3. Place that the ionization chamber in the horizontal position. For this work, we used a 31002 (equivalent to 31010 ) cylindrical ionization chamber calibrated in air kerma.

2.4. Pre-irradiate the ionization chamber for $5 \mathrm{~min}$ and measure background (this step can be performed without a collimator).

2.5. Perform 10 measurements of 1 min each in charge collection mode corresponding to the $M_{\text {raw }}$ value (in coulombs). 
2.6. Take the temperature and pressure with suitable calibrated equipment placed inside the irradiation enclosure in our case (if it is not possible place it near to the experiment). Correct the $\mathrm{M}_{\text {raw }}$ reading on the electrometer by the temperature and pressure correction factor given by

$$
K_{T, P}=\left(\frac{T+273.2}{T_{\text {ref }}+273.2}\right) \times\left(\frac{P_{r e f}}{P}\right)
$$

where: $\mathrm{T}\left({ }^{\circ} \mathrm{C}\right)$ and $\mathrm{P}(\mathrm{hPa})$ are the actual temperature and pressure, respectively. $\mathrm{T}_{\text {ref }}$ and $\mathrm{P}$ ref are the reference temperature and pressure when the ionization was calibrated by standards laboratory. The pressure and temperature must be measured with calibrated instruments.

The obtained value in charge mode is the average reference value $\mathrm{M}$ (in coulombs).

NOTE: This step is not strictly necessary for HVL measurement, but it is recommended.

2.7. Place an attenuator of certain thickness above the diaphragm. The HVL set is composed of foils with different thicknesses $(0.02,0.05,0.1,0.2,0.5,1,2,5$ and $10 \mathrm{~mm}$ of copper) with a dimension allowing to cover the entire beam ( $80 \times 80 \mathrm{~mm}$ here).

2.8. Take a measurement of $1 \mathrm{~min}$ ( $\mathrm{M}_{\text {raw }}$ corrected by the $\mathrm{K}_{\mathrm{T}, \mathrm{P}}$ as described before).

2.8.1. If the dose rate is divided by a factor of 2 with respect to the starting value, the HVL value is found. Take 5 measurements of $1 \mathrm{~min}$ to estimate the average dose rate.

2.8.2. If the dose rate is not divided by a factor of 2 with respect to the starting value, increase or decrease the attenuator thickness and take another measurement. Adjust the thickness of the attenuator as necessary.

2.9. Once the thickness of the attenuator that decreases the intensity of the beam by a factor two is found, take 5 measurements of 1 min to confirm the HVL.

NOTE: In most cases, the exact thickness of attenuator cannot be found from the foils available. In this case, proceed by bisection and interpolate the HVL.

\section{Evaluation of the irradiation field (no dose estimation)}

3.1. Place an EBT3 film on the support used for irradiation.

3.2. Irradiate this film to obtain a well-marked irradiation field (at least 2 Gy).

\subsection{Scan the EBT3 film using a dedicated scanner.}

3.4. Plot the dose profile using Image J by using the Analyze and then plot Profile option (Figure 2)

Page 4 of 11 
3.5. Determine the size of irradiation field usage for irradiation (homogeneous area, excluding penumbra regions, see figure 2)

3.6. Make marks on the support used for irradiation to ensure that the cell container is in the right position

NOTE: In this step, the size of the irradiation field is determined, and the dose is not estimated. The complete procedure for film reading and analysis is given in Section 5. Also, take margins in order to avoid errors due to the cell container positioning.

\section{Dose rate measurement with ionization chamber}

4.1. Take the cell container and break a little part on the side or on the bottom (depending on the particular container and ionization chamber used) to be able to place the ionization chamber inside (Figure 3, upper section) or below (Figure 3, lower section) the container. Examples are given in Figure 3 with different ionization chambers (cylindrical or plane parallel) and cell containers. In this case, a T25 flask was used (Figure 3, red box).

NOTE: a soldering iron or heated scalpel is a good alternative to make holes in plastic ware

4.2. Place the container inside the enclosure on the support used for irradiation (carbon plate here).

4.3. Place the ionization chamber in the container (Figure 3, red box), in the correct position and connect it to the electrometer

4.4. Ensure that all irradiation parameters listed in Section 1 are correct (high voltage, intensity, additional filtrations, source sample distance etc.)

4.5. Pre-irradiate the ionization chamber for $5 \mathrm{~min}$ and perform the zeroing of the electrometer

4.6. Take 10 measurements of $1 \mathrm{~min}$ to determine the average dose rate in air kerma (Gy.min $\left.{ }^{1}\right)$. The determination of the dose rate in $\mathrm{K}_{\text {air }}$ is calculated by:

Where:

$$
K_{\text {air }}=M \times N_{\text {Kair }} \times K_{Q}
$$

$209 M$ is reading of the dosemeter corrected by temperature, pressure, polarity effect, ion recombination and electrometer calibration.

$N_{K a i r}$ and $K_{q}$ are the calibration and correction factors for radiation quality, whose values are specific to each ionization chamber.

\section{Measurement of cell culture media attenuation and scattering}


NOTE: Handle EBT3 films with gloves throughout the procedure.

\subsection{Preparation of the experiment}

5.1.1. Cut small pieces of EBT3 films at least $24 \mathrm{~h}$ before irradiation.

5.1.2. Determine the size of the films as a function of the cell container used for radiobiology experiments ( $4 \times 4 \mathrm{~cm}$ for a T25 flask, for example).

\subsubsection{Cut two sets of radiochromic films must be cut:}

5.1.3.1. One set for the calibration curves composed of three pieces of EBT3 radiochromic film by dose or time point. Nine points in total for this work.

5.1.3.2. One set for the quantification of the cell culture media attenuation, also three pieces per point

5.1.4. Number all the films for identification (upper right corner here) and scan them on the same position on the scanner

\subsubsection{Keep the films away from light}

5.1.6. Prepare the cell container used for the EBT3 film measurements and, if necessary, cut a part to put the film inside (an example with a T25 is given in Figure 4)

\subsection{Dose rate estimation}

5.2.1. Measure the dose rate for the configuration as described in the previous section

5.2.2. Keep this configuration in place for the irradiation of the EBT3 radiochromic films and use the same type of cell container

\subsection{Construction of the calibration curve}

5.3.1. Take the pre-cut EBT3 films for the calibration curve

5.3.2. Three pieces are not irradiated (OGy)

5.3.3. Place the first film inside the cell container, in the same configuration as for cell irradiation

5.3.4. Irradiate it to obtain the first dose points 
5.3.5. Repeat this operation to obtain three pieces of EBT3 films irradiated with the same dose

5.3.6. Perform this for each dose point (Nine dose points in this work $(0,0.25,0.5,0.75,1,1.5$, 2, 2.5 and $3 \mathrm{~Gy}$ ) as illustrated in Figure 5)

5.4. Evaluation of the attenuation of cell culture media and scattering

5.4.1. Chose the same irradiation time for all irradiations ( 60 seconds, for example)

5.4.2. Irradiate three pieces of EBT3 films in the container without water

5.4.3. Irradiate three pieces of EBT3 films in the container with water as follows:

5.4.3.1. Place the film inside the container

5.4.3.2. Fill the container with the exact quantity of water to represent the cell culture media ( $5 \mathrm{~mL}$ here). Use small pieces of tape if the films do not remain submerged properly.

5.4.3.3. Place the cell container inside the enclosure and ensure that the film is correctly immersed

5.4.3.4. When the irradiation is complete, take the EBT3 films, dry them with absorbent paper, and store them away from light

\section{Reading of EBT3 radiochromic films}

\subsection{Read EBT3 films at least $24 \mathrm{~h}$ after irradiation}

6.2. Scan the films on an dedicated scanner

6.3. Set the scanner parameters as: 48 bit red-green-blue tiff format, $150 \mathrm{dpi}$ in transmission mode and no image correction

6.4. Perform warm up of the scanner by following the steps below

6.4.1. Place a non-irradiated film on the scanner

6.4.2. Launch a preview of the scan

6.4.3. Launch a timer and wait for 30 seconds

6.4.4. Launch the scan

Page 7 of 11 
6.4.6. At the same time, register the scan, open the image with ImageJ, trace a square ROI (always the same size and in the same position), and take a measurement of the average red pixel level of the area

\subsubsection{At the end of the 90 seconds, repeat the procedure from Step two (without touching the} film inside the scanner)

6.4.8. Repeat this at least 30 times to warm up and stabilize the scanner (no variations in the average red pixel level of the area selected on the non-irradiated films). If the scanner, i.e. the average red pixel value, is not stabilized, continue the procedure.

\subsection{Scanning of the EBT3 films}

6.5.1. Place the first film in the center of the scanner bed. You should delimitate an area to always place the film in the same place and in the same orientation

\subsubsection{Launch a preview of the scan}

\subsubsection{Launch a timer and wait for 30 seconds}

\subsubsection{Launch the scan}

6.5.5. At the end of the scan, launch a timer, and wait for 90 seconds. During these 90 seconds change the EBT3 film

Note: An analysis of the EBT3 radiochromic films was performed using a self-programmed C++ program. Different methods can be used for the EBT3 film analysis, such as the red channel method or the three channels method ${ }^{14,15}$. In this case, we have used the red channel method with no background subtraction, and the images were converted to optical densities and then to the dose using our program. As this method is already well defined, our $\mathrm{C}++$ program was not included here. Moreover, dedicated software ${ }^{16}$ can also be used for EBT3 film analysis.

\section{Determination of the dose rate at the level of the cell monolayer}

7.1. Convert the average dose rate obtained with the ionization chamber corrected by the attenuation and scattering of the cell culture media $(\mathrm{K})$ to the water kerma using the ratio of the mean mass energy absorption coefficient for water to air evaluated over the photon fluence spectrum $\left(\mu_{\mathrm{en}} / \rho\right)$.

$$
K_{\text {water }}=K \times\left[\left(\frac{\bar{\mu}_{e n}}{\rho}\right)_{\text {air }}^{w}\right]_{\text {air }}
$$

A dedicated software ${ }^{17}$ was used to calculate the photon energy spectrum in air with no Page 8 of 11 
phantom, and we used the NIST table ${ }^{18}$ to calculate the mean mass energy absorption coefficient.

\section{REPRESENTATIVE RESULTS:}

In this work, we used a platform dedicated to small animal irradiation ${ }^{19}$, however this platform can be used to irradiate other types of samples such as cells. The irradiation source is a Varian Xray tube (NDI-225-22, NDI, Washington, DC) having an inherent filtration of $0.8 \mathrm{~mm}$ of beryllium, a large focal sport size of $3 \mathrm{~mm}$, a high voltage range about 30 to $225 \mathrm{kV}$ and a maximal intensity of $30 \mathrm{~mA}$.

The parameters used for this study are reported in Table 1. We have chosen to show an example of the use of this protocol for cell irradiation in a T25 flask with $5 \mathrm{~mL}$ of cell culture media.

Half value layer:

Table 2 reports the measurements performed to estimate the attenuator thickness needed to decrease the intensity of the beam by a factor of two. For this, 10 reference measurements were taken to estimate the average $\mathrm{M}_{\text {raw }}$ reading on the electrometer (in coulombs), corrected by the temperature and pressure correction factor $\left(\mathrm{K}_{\mathrm{T}, \mathrm{P}}\right)$.

Different thickness of attenuator were then tested to find the thickness that decreased the beam intensity by a factor of two. When this thickness was found, five measurements were taken to evaluate the average $\mathrm{M}_{\text {raw }}$ value corrected by $\mathrm{K}_{\mathrm{T}, \mathrm{P}}$.

For this configuration, a half value layer of $0.667 \mathrm{~mm}$ of copper was found. From the HVL measurement, we can calculate the effective energy of the beam, which is about $69 \mathrm{keV}$ in our case.

\section{Dose rate measurement:}

Before to these measurements, an EBT3 film was irradiated in order to determine the surface on which the irradiation field is homogeneous, allowing us to correctly place the cell container. This area is about $10 \times 10 \mathrm{~cm}^{2}$ excluding penumbra regions shown by dotted lines in figure 2 . Then, dose rate measurement was performed using a 31002 (equivalent to 31010) cylindrical ionization chamber calibrated in air kerma. For this configuration, with an open field irradiation field at 35 $\mathrm{cm}$ to the source in a T25 cell container placed on a carbon plate, the dose rate was about 0.626 Gy. $\min ^{-1}$ in Kair.

To determine the exact dose on cells, the measured $\mathrm{K}_{\text {air }}$ was converted in water kerma. Figure 5 shows the $\mathrm{X}$-rays energy spectrum obtained with dedicated software ${ }^{17}$. From this energy spectrum and the NIST table, we can convert the dose rate in $K_{\text {air }}$ to $K_{\text {water, }}$ which was 0.659 Gy. $\min ^{-1}$.

385

The overall uncertainty of the absolute dose rate measurement was about $3 \%$ at a $95 \%$ confidence level. 
Cell culture media attenuation and scattering:

For the quantification of cell culture media attenuation and scattering, dosimetry measurements with EBT3 radiochromic films were performed at room temperature. From the measurement with the ionization chamber, the dose rate was determined. Calibration films were then irradiated at the same position. EBT3 radiochromic films were calibrated between 0 and 3 Gy with 0.25 Gy steps between 0 and $1 \mathrm{~Gy}$ and $0.5 \mathrm{~Gy}$ steps between 1 and $3 \mathrm{~Gy}$ (nine dose points to construct the calibration curve) as shown in Figure 6 . The dose points were fitted with a $4^{\text {th }}$ degree polynomial curve. The EBT3 films were then irradiated with and without the exact quantity of cell culture media inside the cell container to evaluate the attenuation and the scattering due to the cell culture media. For this configuration, the attenuation of the cell culture media was about $1.5 \%$.

The overall uncertainty of the EBT3 film measurements was about $4 \%$ at a $95 \%$ confidence level.

\section{Routine measurements:}

Before performing the cell irradiations, the dose rate was measured each time in the same container used for irradiation. Thus, we used the daily dose rate to estimate our irradiation time. If we closely follow the protocol and do not change any parameters, the HVL measurement and the attenuation due to the cell culture media do not need to be repeated. As an example, the table used for our daily measurement is given in Table 3.

\section{FIGURE AND TABLE LEGENDS:}

Figure 1: Scheme of the configuration take in place on the SARRP enclosure for HVL measurements.

Figure 2: Evaluation of the irradiation field size. Dose profile obtained at $35 \mathrm{~cm}$ to the source without collimator. Dotted lines show the area considered for the irradiation.

Figure 3: Photograph of cell containers with the ionization chamber for dose rate measurement. Upper part: example for measurement with a 31002 cylindrical ionization chamber. Lower part: example for measurement with a TM23342 ionization chamber.

Figure 4: Photograph of the T25 used for the measurement of the cell culture media attenuation. The upper part of the T25 was cut out to be able to place the film inside the flask.

Figure 5: Simulated energy spectra for a $220 \mathrm{kV}$ high voltage with $0.8 \mathrm{~mm}$ of $\mathrm{Be}$ and $0.15 \mathrm{~mm}$ of $\mathrm{Cu}$ filtrations ${ }^{17}$.

Figure 6: EBT3 films irradiated to construct the calibration curve and the corresponding calibration curve.

Table1: List of the configuration parameters.

Page 10 of 11 
Table 2: Measurement for the Half Value Layer determination.

Table 3: Daily dose rate measurements for cell irradiation.

\section{DISCUSSION:}

This work presents the protocol used and implemented for cell irradiations using low energy $X-$ rays facility. Nowadays, many radiobiology experiments are performed with this type of irradiator as they are easy to use, cost effective and with very few radioprotection constraints, compared to cobalt source for example. Although these set-ups have many advantages, as they use a low $X$-ray energy source, a modification of only one irradiation parameter can significantly impact the dosimetry. Several studies have already highlighted the importance of dosimetry standards and protocols for radiobiology studies $2,5,20,21$. Even though several protocols have already been well defined in the literature ${ }^{1,5}$, we decided to develop a new protocol to perform dosimetry measurements to simulate real cell irradiation conditions as much as possible and take in account all the parameters that can influence the physical dose, especially for low energy X-rays ${ }^{21,22}$. Thus, we have chosen to implement a stringent protocol to minimize uncertainties. To this end, irradiation parameters were set (Table1). Three steps are then necessary: i) determination of the beam quality index, ii) measurement of the absolute dose rate with an ionization chamber and iii) measurement of the attenuation and scattering due to the cell culture medium with EBT3 radiochromic films.

The beam quality index corresponded to the voltage-half value layer (HVL) couple used to characterize low energy $X$-rays beams. The HVL is a practical indicator to describe poly energetic radiation and is defined as the thickness of an attenuator (usually copper or aluminum) to reduce the air kerma dose rate by a factor of two from the original value. HVL measurements were performed using the following recommendations of the AAPM protocol for a 40-300 kV X-ray beam ${ }^{10}$. However, some adaptations had to be made because in the irradiator enclosure it is not possible to achieve a distance a 1 meter between the source and the ionization chamber. Therefore, in the present work, we used a distance of $58 \mathrm{~cm}$ between the source and the detector for HVL measurements, as illustrated in Figure 1 . We decided to let $25 \mathrm{~cm}$ after the ionization chamber because a lot of electronical material, support and metallic element are present in the bottom of the enclosure to limit the backscatter effect of these elements. Measurement of the $\mathrm{HVL}$ is one of the critical aspects of this protocol. Indeed, for many X-rays irradiators, the inside of enclosures is very restricted and these are not the optimal conditions to perform the measurements or it becomes impossible. Although experimental measurements are the best way to evaluate the HVL, when these measurements were too difficult or even impossible to perform, dedicated software ${ }^{17}$ can be used to provide a good estimation for the HVL or a Monte Carlo simulation can be used ${ }^{23}$. In the present work, we used a dedicated software to obtain the X-ray energy spectrum (Figure 5). We were also able to compare the measured and calculated HVL, which was the same, and to also compare the effective energy. 
472 For dosimetry measurements, we then chose to simulate real cell irradiation conditions as much

473 as possible. For this, we directly performed the absolute dose rate measurements with the 474 ionization chamber inside the cell container used for cells irradiation (Figure 3). However, as we 475 used a cylindrical ionization chamber calibrated for beams over $100 \mathrm{kV}$, we were not exactly at the same position as the cells because of the thickness of the ionization chamber. For lower beams (15-70kV), where plan parallel chamber can be used, we can be even closer to the real cell irradiation conditions. Then, relative dosimetry measurements were performed to evaluate the attenuation and the scattering due to the cell culture medium. The results presented on this work do not highlight a significant variation in the dose deposited with or without the exact quantity of cell culture media as we used a voltage of $220 \mathrm{kV}$, an additional filtration of $0.15 \mathrm{~mm}$ of $\mathrm{Cu}$ and we only had $5 \mathrm{~mL}$ of cell culture medium. However, in our previous study ${ }^{21}$ carried out at $80 \mathrm{kV}$, we pointed out that a variation of the cell culture media and filtration significantly impacts the physical dose, up to $40 \%$ compared to the reference configuration when we used a $1 \mathrm{~mm}$ aluminum filtration. This impact was also demonstrated in terms of biological effects by measuring the surviving cell fraction using a clonogenic assay ${ }^{21,24}$. Thus, depending on the voltage, additional filtration, the container and quantity of cell culture media, the dose deposited on the cells can be different if the protocol is not closely followed for all irradiations.

Consequently, a dedicated dosimetry should be set up for all cell irradiation configurations. Although this is restrictive and the modification of only a single parameter requires the implementation of a new configuration, we have decided to make this choice to be as close as possible to the real cell irradiation conditions. This requires a close collaboration between the physicists and the radiobiologist in order to set up the best design for the configuration. At our institute, a dozen protocols were established on our platform for a voltage range of 40 to $220 \mathrm{kV}$ for which T25, T75, 6- to 96-plate wells or petri dishes can be irradiated.

Although this protocol seems quite long to implement, once the configuration is established, the only measurement to be taken on the day of irradiation is measurement of the dose rate with the ionization chamber inside the cell container. This measurement is also a quality control that enables us to ensure that the dose rate is as expected.

To ensure the reproducibility of radiobiological studies, and to be able to compare and interpret experiments, it is important is to rigorously follow established protocols and report all the dosimetry and configuration aspects, particularly for facilities using low or medium energy $X$-rays. The new protocol proposed here is for cell irradiations, applicable to many X-rays facilities, and takes into account all parameters influencing the dosimetry and provides a better estimation of the actual dose delivered to cells.

511 The authors have nothing to disclose.

\section{REFERENCES}


5151 Zoetelief, J., Broerse, J. J. \& Davies, R. W. Protocol for X-ray dosimetry EULEP. Report No. 516 Report EUR 9507, (Commission of the European Communities, 1985).

5172 Zoetelief, J. et al. Protocol for X-ray dosimetry in radiobiology. International Journal of 518 Radiation Biology. 77 (7), 817-835, doi:10.1080/09553000110050605, (2001).

5193 Zoetelief, J. \& Jansen, J. T. Calculated energy response correction factors for LiF 520 thermoluminescent dosemeters employed in the seventh EULEP dosimetry intercomparison. 521 Physics in Medicine and Biology. 42 (8), 1491-1504 (1997).

5224 Coleman, C. N. et al. Education and training for radiation scientists: radiation research 523 program and American Society of Therapeutic Radiology and Oncology Workshop, Bethesda, 524 Maryland, May 12-14, 2003. Radiation Research. 160 (6), 729-737 (2003).

5255 Desrosiers, M. et al. The Importance of Dosimetry Standardization in Radiobiology. J Res 526 Natl Inst Stand Technol. 118 403-418, doi:10.6028/jres.118.021, (2013).

5276 (DIN), D. I. f. N. Klinische Dosimetrie: Teil 4. Anwendung von Röntgenstrahlen mit 528 Röhrenspannungen von 10 bis $100 \mathrm{kV}$ in der Strahlentherapie und in der Weichteildianostik. 529 Report No. DIN 6809, DIN 6809 (1988).

5307 (DIN), D. I. f. N. Klinische Dosimetrie: Teil 5: Anwendung von Röntgenstrahlen mit 531 Röhrenspannungen von 100 bis 400 kV in der Strahlentherapie. Report No. DIN 6809-5, DIN 68095326805 (1996).

5338 (NCS), N. C. v. S. Dosimetry of low and medium energy x-rays: A code of practice for use 534 in radiotherapy and radiobiology. Report No. Report No. 10, (1997).

5359 IAEA. Absorbed Dose Determination in External Beam Radiotherapy. (INTERNATIONAL 536 ATOMIC ENERGY AGENCY, 2000).

$53710 \mathrm{Ma}, \mathrm{C}$. M. et al. AAPM protocol for 40-300 kV x-ray beam dosimetry in radiotherapy and 538 radiobiology. Medical Physics. 28 (6), 868-893, doi:10.1118/1.1374247, (2001).

53911 Peixoto, J. G. \& Andreo, P. Determination of absorbed dose to water in reference 540 conditions for radiotherapy kilovoltage x-rays between 10 and $300 \mathrm{kV}$ : a comparison of the data 541 in the IAEA, IPEMB, DIN and NCS dosimetry protocols. Physics in Medicine and Biology. 45 (3), 542 563-575 (2000).

54312 Pedersen, K. H., Kunugi, K. A., Hammer, C. G., Culberson, W. S. \& DeWerd, L. A. Radiation 544 Biology Irradiator Dose Verification Survey. Radiation Research. 185 (2), 163-168, 545 doi:10.1667/RR14155.1, (2016).

54613 Draeger, E. et al. A Dose of Reality: How 20 Years of Incomplete Physics and Dosimetry 547 Reporting in Radiobiology Studies May Have Contributed to the Reproducibility Crisis. 548 International Journal of Radiation Oncology Biology Physics. 106 (2), 243-252, 549 doi:10.1016/j.ijrobp.2019.06.2545, (2020).

55014 Devic, S. et al. Precise radiochromic film dosimetry using a flat-bed document scanner. 551 Medical Physics. 32 (7), 2245-2253, doi:10.1118/1.1929253, (2005).

55215 Micke, A., Lewis, D. F. \& Yu, X. Multichannel film dosimetry with nonuniformity correction. 553 Medical Physics. 38 (5), 2523-2534, doi:10.1118/1.3576105, (2011).

$55416 \quad$ http://www.gafchromic.com/filmqa-software/filmqapro/index.asp.

55517 Poludniowski, G., Landry, G., DeBlois, F., Evans, P. M. \& Verhaegen, F. SpekCalc: a program 556 to calculate photon spectra from tungsten anode x-ray tubes. Physics in Medicine and Biology. 55754 (19), N433-438, doi:10.1088/0031-9155/54/19/N01, (2009). 
55818 Hubbell, J. H. \& Seltzer, S. M. X-Ray Mass Attenuation Coefficients - Tables of X-Ray Mass 559 Attenuation Coefficients and Mass Energy-Absorption Coefficients $1 \mathrm{keV}$ to $20 \mathrm{MeV}$ for Elements $560 \quad Z=1$ to 92 and 48 Additional Substances of Dosimetric Interest (Version 1.4). NIST Standard 561 Reference Database 126. doi:10.18434/T4D01F, (1995).

56219 Wong, J. et al. High-resolution, small animal radiation research platform with x-ray 563 tomographic guidance capabilities. International Journal of Radiation Oncology Biology Physics. 56471 (5), 1591-1599, doi:10.1016/j.ijrobp.2008.04.025, (2008).

$56520 \quad$ Trompier, F. et al. Investigation of the influence of calibration practices on cytogenetic 566 laboratory performance for dose estimation. International Journal of Radiation Biology. 1-9, 567 doi:10.1080/09553002.2016.1213455, (2016).

56821 Dos Santos, M. et al. Importance of dosimetry protocol for cell irradiation on a low X-rays 569 facility and consequences for the biological response. International Journal of Radiation Biology. 570 1-29, doi:10.1080/09553002.2018.1466205, (2018).

57122 Noblet, C. et al. Underestimation of dose delivery in preclinical irradiation due to 572 scattering conditions. Physica Medica. 30 (1), 63-68, doi:10.1016/j.ejmp.2013.03.001, (2014).

$57323 \quad$ Paixao, L. et al. Monte Carlo derivation of filtered tungsten anode X-ray spectra for dose 574 computation in digital mammography. Radiologia Brasileira. 48 (6), 363-367, doi:10.1590/0100$5753984.2014 .0108,(2015)$. 


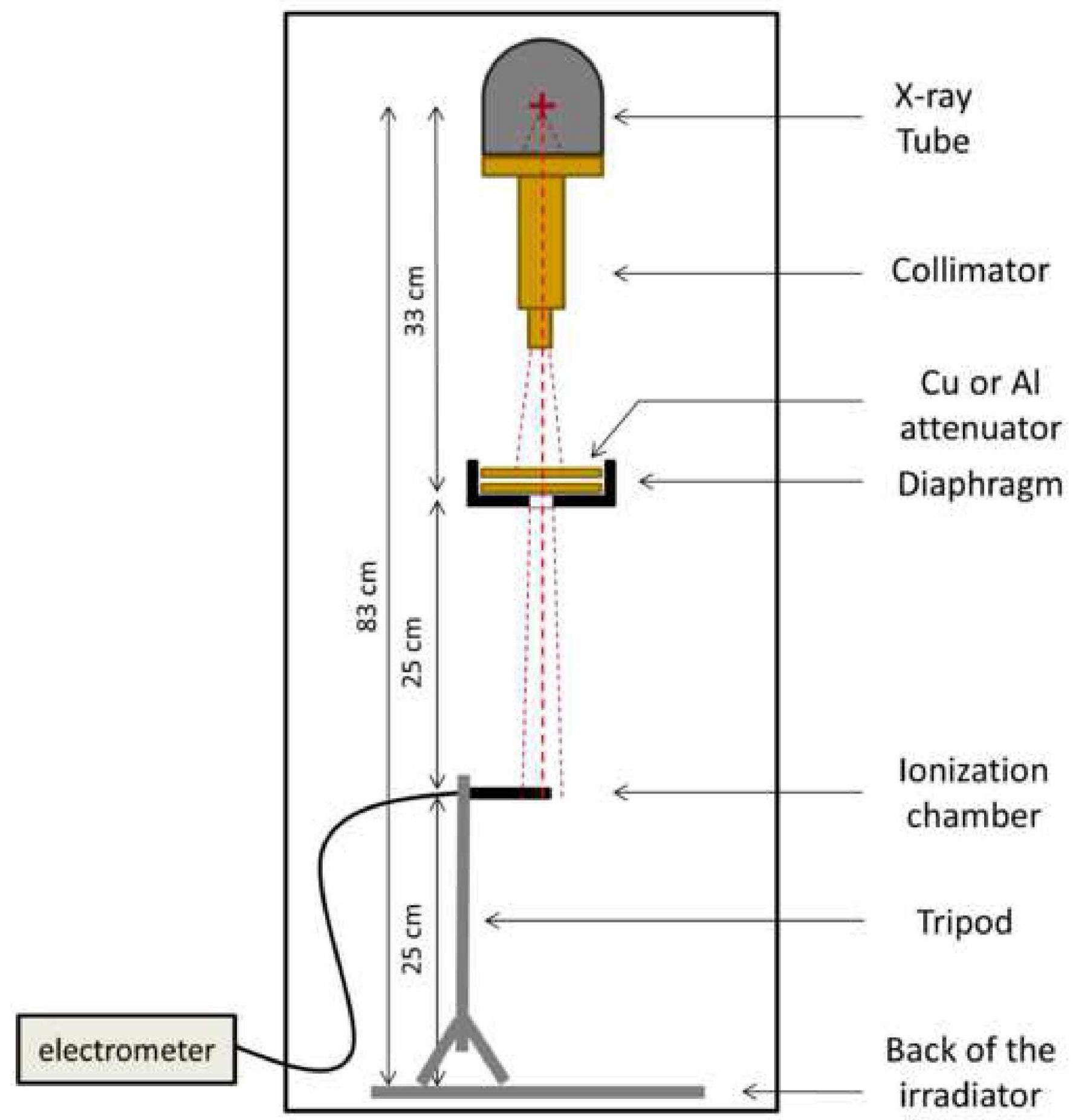




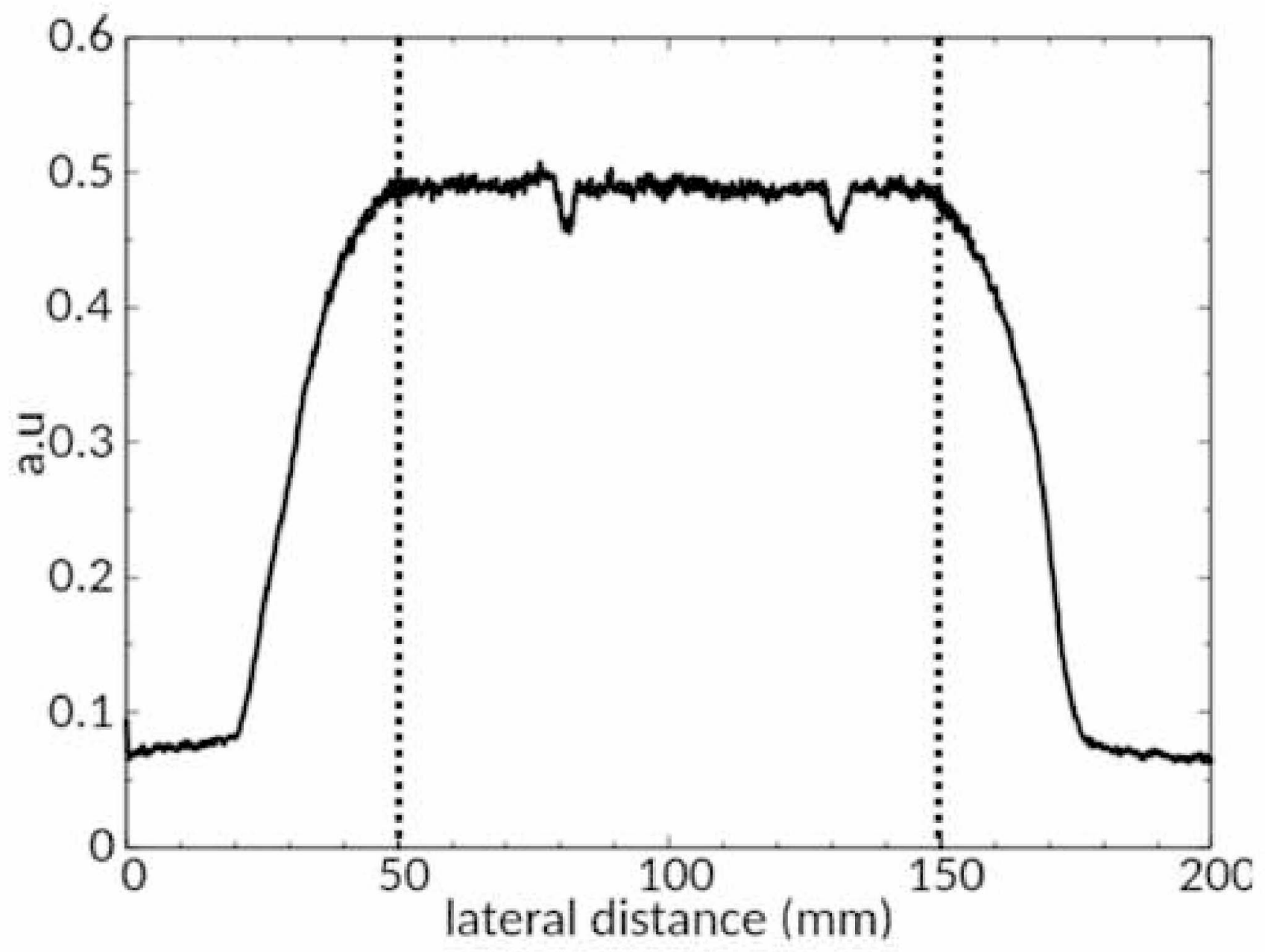



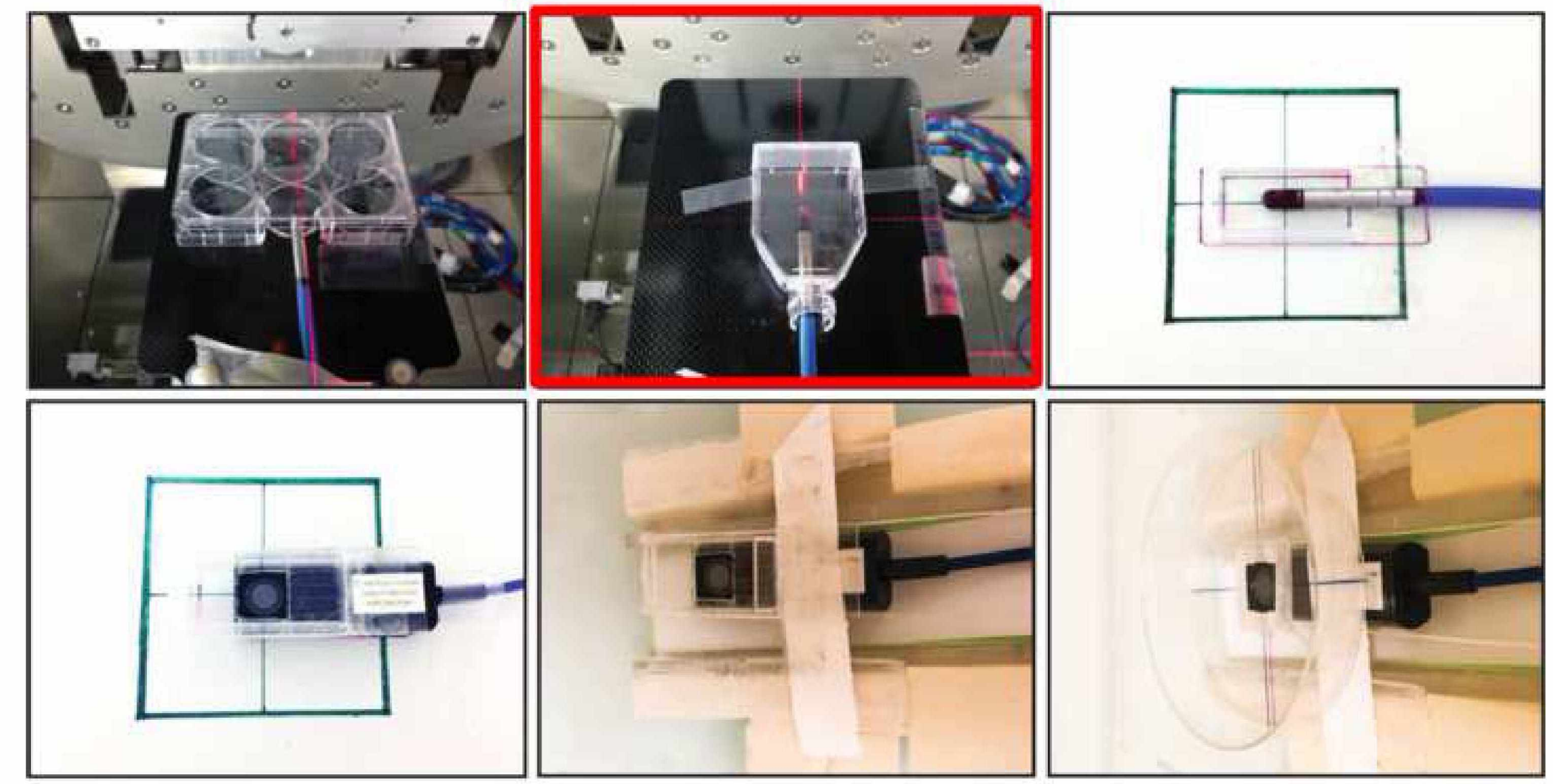


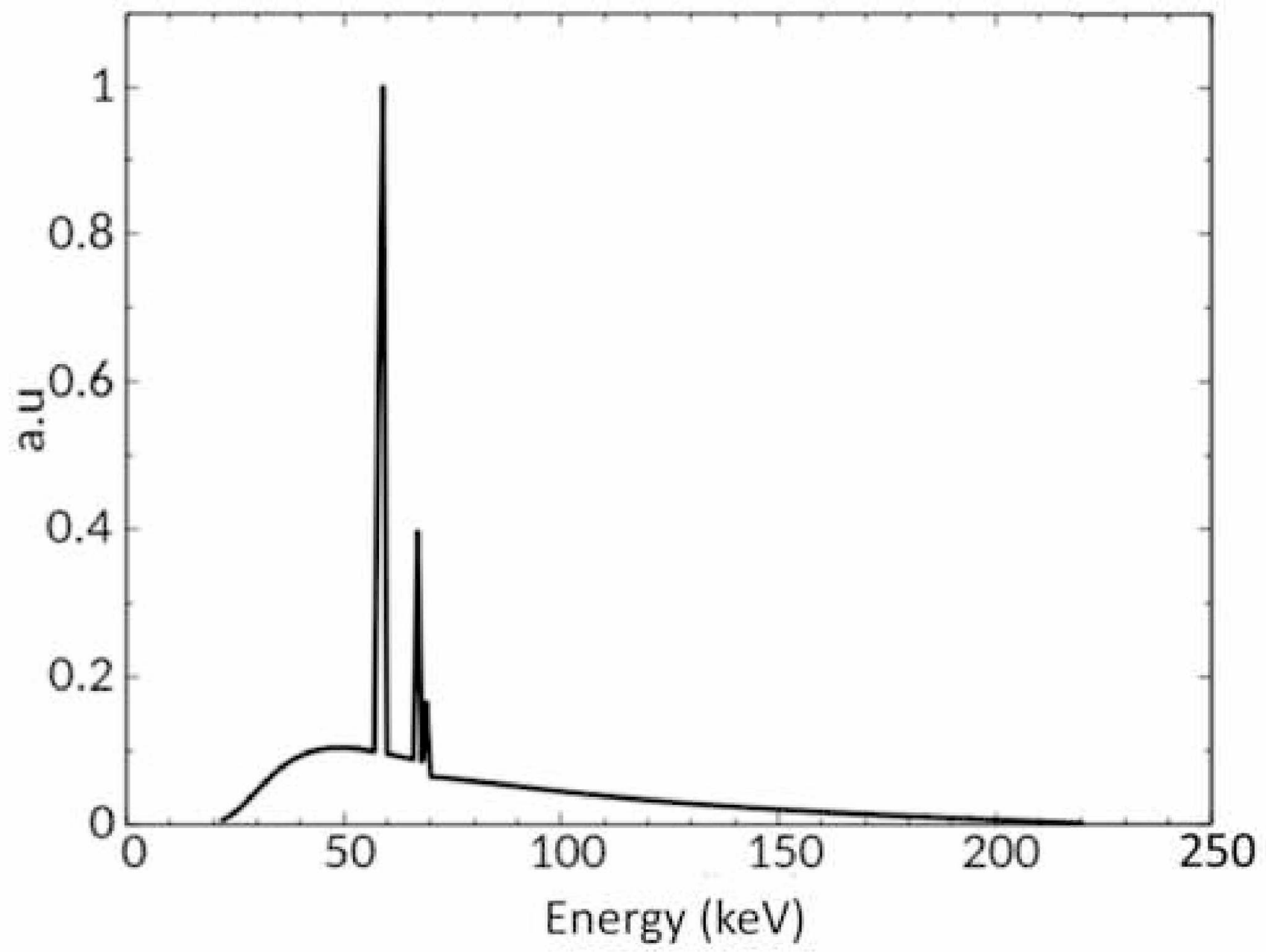



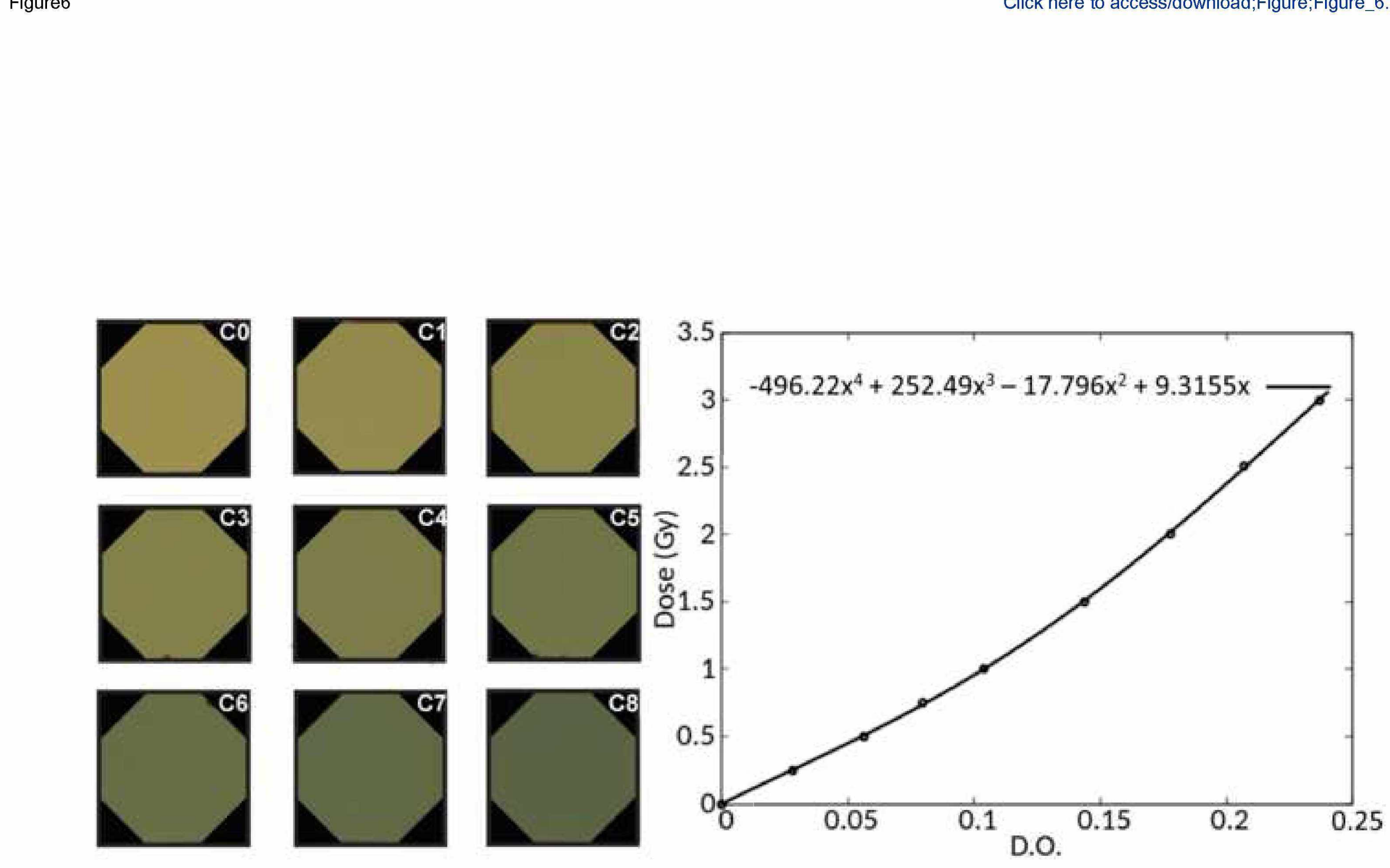


\begin{tabular}{|c|c|}
\hline High voltage (kV) & 220 \\
\hline Intensity (mA) & 3 \\
\hline Filtrations (inherent and additional) & $0.8 \mathrm{~mm}$ of $\mathrm{Be}+0.15 \mathrm{~mm} \mathrm{Cu}$ \\
\hline Half value layer $(\mathrm{mm} \mathrm{Cu})$ & Determined below \\
\hline Effective energy (keV) & Determined below \\
\hline Detector used & $\begin{array}{l}\text { Cylindrical ionization chamber }+ \text { EBT3 radiochromic } \\
\text { films }\end{array}$ \\
\hline Source sample distance & $35 \mathrm{~cm}$ \\
\hline Irradiation field (shape, size, geometry) & Open field (no collimator), square, $20 \times 20 \mathrm{~cm}$ \\
\hline Dosimetry quantity & Kair and Kwater \\
\hline Dosimetry method & As described on the protocol section \\
\hline Cell container & $\mathrm{T} 25$ \\
\hline Quantity of cell culture media & $5 \mathrm{ml}$ \\
\hline Dose rate (Gy/min) & Determined below \\
\hline
\end{tabular}




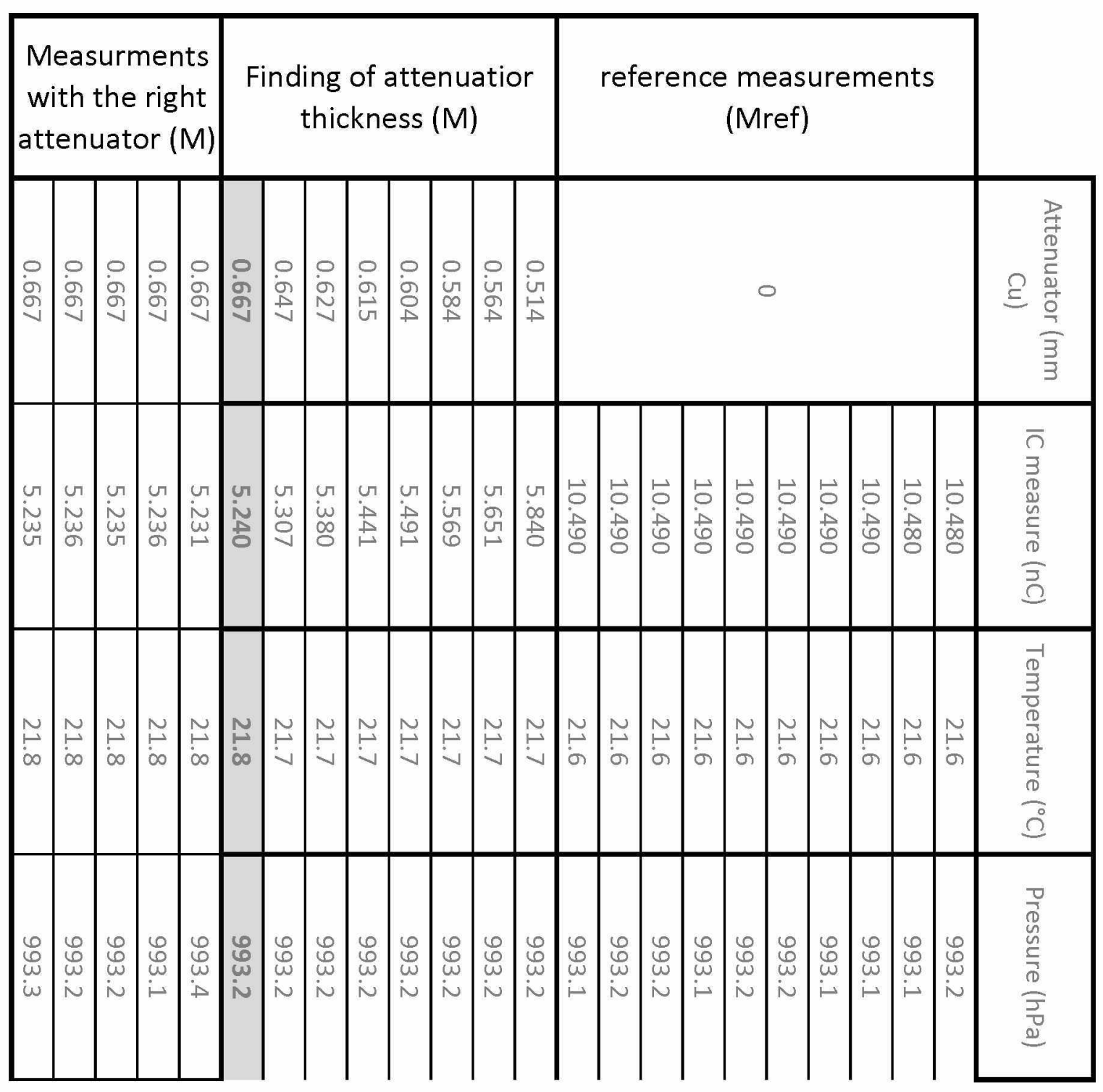

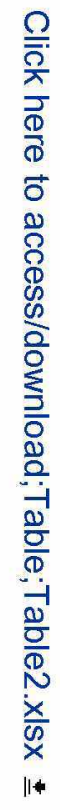




\begin{tabular}{|c|c|c|c|}
\hline $\mathrm{k}_{\mathrm{T} . \mathrm{P}}$ & $\begin{array}{c}\text { IC measure } \\
\text { corrected by } k_{\mathrm{T}, \mathrm{P}} \\
(\mathrm{nC})\end{array}$ & $\begin{array}{c}\text { Corrected Mean } \\
\text { value }(\mathrm{nC})\end{array}$ & ST deviation \\
\hline 1.026 & 10.752 & \multirow{10}{*}{10.761} & \multirow{10}{*}{0.005} \\
\hline 1.026 & 10.752 & & \\
\hline 1.026 & 10.763 & & \\
\hline 1.026 & 10.763 & & \\
\hline 1.026 & 10.763 & & \\
\hline 1.026 & 10.763 & & \\
\hline 1.026 & 10.763 & & \\
\hline 1.026 & 10.763 & & \\
\hline 1.026 & 10.763 & & \\
\hline 1.026 & 10.763 & & \\
\hline 1.026 & 5.992 & - & - \\
\hline 1.026 & 5.798 & - & - \\
\hline 1.026 & 5.714 & - & - \\
\hline 1.026 & 5.634 & - & - \\
\hline 1.026 & 5.582 & - & - \\
\hline 1.026 & 5.520 & - & - \\
\hline 1.026 & 5.445 & - & - \\
\hline 1.026 & 5.376 & - & - \\
\hline 1.026 & 5.368 & \multirow{5}{*}{5.373} & \multirow{5}{*}{0.003} \\
\hline 1.026 & 5.375 & & \\
\hline 1.026 & 5.373 & & \\
\hline 1.026 & 5.374 & & \\
\hline 1.026 & 5.373 & & \\
\hline
\end{tabular}




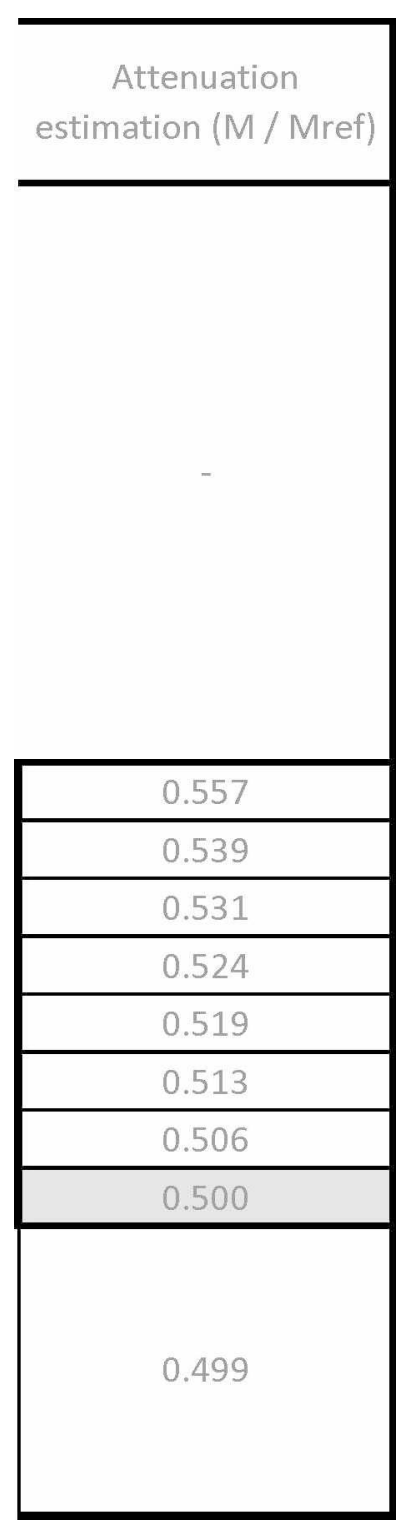




\begin{tabular}{|c|c|c|c|}
\hline IC measure $(\mathrm{nC})$ & Temperature $\left({ }^{\circ} \mathrm{C}\right)$ & Pressure $(\mathrm{hPa})$ & $\mathrm{k}_{\text {T.P }}$ \\
\hline 2.495 & 22.3 & 1001 & 1.020 \\
\hline 2.496 & 22.3 & 1001 & 1.020 \\
\hline 2.497 & 22.3 & 1001 & 1.020 \\
\hline 2.498 & 22.3 & 1001 & 1.020 \\
\hline 2.496 & 22.3 & 1001 & 1.020 \\
\hline 2.495 & 22.3 & 1000.9 & 1.020 \\
\hline 2.494 & 22.3 & 1000.9 & 1.020 \\
\hline 2.495 & 22.3 & 1000.9 & 1.020 \\
\hline 2.496 & 22.3 & 1000.9 & 1.020 \\
\hline 2.496 & 22.3 & 1000.9 & 1.020 \\
\hline
\end{tabular}




\begin{tabular}{|c|c|c|c|c|}
\hline $\begin{array}{c}\text { IC measure } \\
\text { corrected by } k_{\text {T.P }} \\
(\mathrm{nC})\end{array}$ & $\begin{array}{l}\text { Corrected Mean } \\
\text { value by } k_{\text {T.P }}(\mathrm{nC})\end{array}$ & ST deviation & $\begin{array}{l}\text { Corrected mean } \\
\text { value by all } \\
\text { correction factors }\end{array}$ & $\begin{array}{l}\text { Dose rate in air } \\
\operatorname{kerm}(G y / m i n)\end{array}$ \\
\hline 2.545 & \multirow{10}{*}{2.546} & \multirow{10}{*}{0.001} & \multirow{10}{*}{2.536} & \multirow{10}{*}{0.626} \\
\hline 2.546 & & & & \\
\hline 2.547 & & & & \\
\hline 2.548 & & & & \\
\hline 2.546 & & & & \\
\hline 2.545 & & & & \\
\hline 2.544 & & & & \\
\hline 2.545 & & & & \\
\hline 2.546 & & & & \\
\hline 2.546 & & & & \\
\hline
\end{tabular}




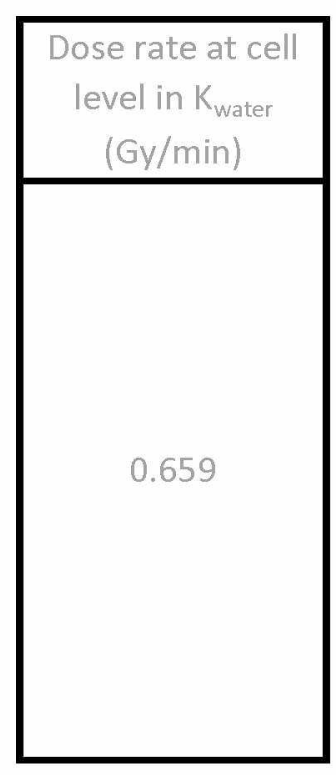




\begin{tabular}{|c|c|c|}
\hline Name of Reagent/ Equipment & Company & Catalog Number \\
\hline 31010 ionization chamber & PTW & $\begin{array}{c}\text { ionization Radiation, } \\
\text { Detectors including code of } \\
\text { practice, catalog 2019/2020, } \\
\text { page 14 }\end{array}$ \\
\hline electrometer UNIDOSEwebline & PTW & online catalog, quote request \\
\hline $\begin{array}{c}\text { temperature and pressure } \\
\text { measurements, Lufft OPUS20 }\end{array}$ & lufft & quote request \\
\hline EBT3 radiochromic films & meditest & quote request \\
\hline HVL material (filter, diaphragm) & PTW & online catalog, page 70, quote \\
& & request \\
\hline scanner for radiochromic films & epson & quote request \\
\hline
\end{tabular}




\begin{tabular}{|c|}
\hline Comments/Description \\
$\frac{\text { https://www.ptwdosimetry.com/fileadmin/user upload/DETECTORS Cat en } 16522900 \quad 1}{\text { 2/blaetterkatalog/index.html?startpage=1\#page } 14}$ \\
\hline$\underline{\text { https://www.ptwdosimetry.com/en/products/unidos- }}$ \\
$\underline{\text { webline/?type=3451\&downloadfile=1593\&cHash=6096ddc2949f8bafe5d556e931e6c865 }}$ \\
\hline$\underline{\text { https://www.lufft.com/products/in-room-measurements-291/opus-20-thip-1983/ }}$ \\
\hline$\underline{\text { https://www.meditest.fr/produit/ebt3-8x10/ }}$ \\
https://www.ptwdosimetry.com/fileadmin/user_upload/Online_Catalog/Radiation \\
_Medicine_Cat_en_58721100_11/blaetterkatalog/index.html\#page_70 \\
\hline Epson V700, seiko Epson corporation, Suwa, Japan
\end{tabular}

